\title{
Analysis Finds $B$ Meson Behaves Itself
}

\author{
A new analysis of Large Hadron Collider data measures rare decays of the \\ $B$ meson that behave according to the standard model.
}

\author{
By Sophia Chen
}

$\prod$

he standard model of particle physics predicts precisely the various decay rates of the $B$ meson, an unstable neutral or charged particle consisting of a bottom antiquark and another quark. By experimentally verifying that the $B$ meson's rare decays conform to theory, particle physicists aim to pinpoint any deviations from predictions that would indicate the existence of new particles or processes beyond the standard model. Now, the Large Hadron Collider beauty (LHCb) Collaboration has released new measurements relating to three rare decay channels that are consistent with theoretical predictions [1, 2]. The result constrains parameters such as the mass of potential Higgs bosons in proposed models beyond the standard model.

The team's eponymous LHCb detector is designed to search for signatures of bottom quarks in proton-proton collisions at the Large Hadron Collider. In this analysis, the researchers used data from two different experimental runs. The center-of-mass collision energies vary among 7, 8, and $13 \mathrm{TeV}$.

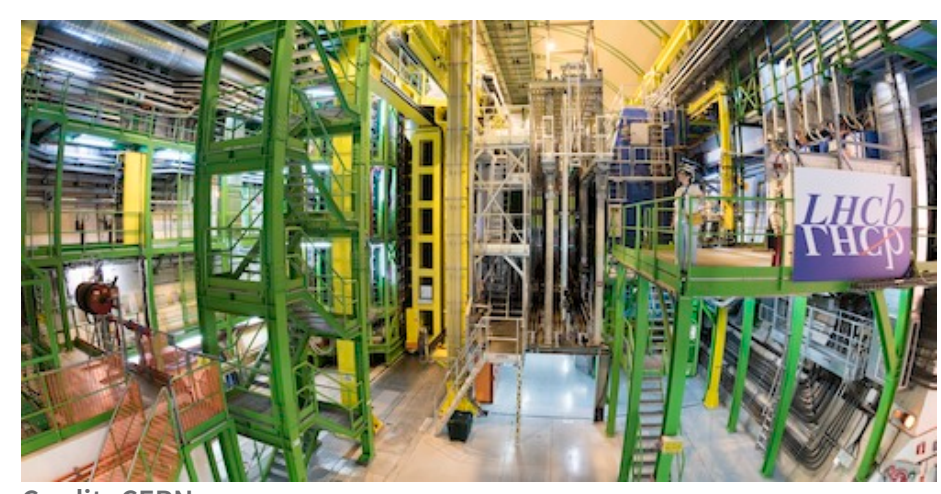

Credit: CERN

The new analyses yielded the most precise measurements yet of three rare decay channels in which the $B$ meson's decay products include two muons. These decays are rare because they require the exchange of multiple virtual particles. The researchers found that the strange $B$ meson, which consists of a bottom antiquark and a strange quark, decays into a pair of muons three times every billion decays, consistent with their previous measurement.

The researchers did not find statistically significant signals of the other two decay channels. Still, they were also able to put upper limits on the likelihood of two processes: With $95 \%$ confidence, the researchers determined that less than 26 in 100 billion neutral $B$ mesons (a bottom quark and a down quark) decay into a pair of muons, and less than 2 in a billion strange $B$ mesons decay into two muons (with a combined mass above $4.9 \mathrm{GeV} / \mathrm{c}^{2}$ ) and a photon. This was also LHCb's first search for the strange $B$ meson decay, which has been difficult to observe because its selection is affected by a larger background. The collaboration plans to collect more data in this region in the LHC's third run, to begin at the end of 2022, to further study how these $B$ meson decay channels could probe new physics.

Sophia Chen is a freelance science writer based in Columbus, Ohio.

\section{REFERENCES}

1. R. Aaij et al., "Analysis of neutral B-meson decays into two muons," Phys. Rev. Lett. 128, 041801 (2022).

2. R. Aaij et al., "Measurement of the $B_{s}^{0} \rightarrow \mu^{+} \mu^{-}$decay properties and search for the $B^{0} \rightarrow \mu^{+} \mu^{-}$and $B_{s}^{0} \rightarrow \mu^{+} \mu^{-} \gamma$ decays," Phys. Rev. D 105, 012010 (2022). 